\title{
A Study of New Nondispersive SH-SAWs in Magnetoelectroelastic Medium of Symmetry Class 6 mm
}

\author{
Aleksey Anatolievich Zakharenko \\ International Institute of Zakharenko Waves (IIZWs), Krasnoyarsk, Russia \\ Email: aazaaz@inbox.ru
}

Received 20 July 2015; accepted 14 September 2015; published 17 September 2015

Copyright (C 2015 by author and Scientific Research Publishing Inc.

This work is licensed under the Creative Commons Attribution International License (CC BY).

http://creativecommons.org/licenses/by/4.0/

\section{Abstract}

Two additional solutions of new shear-horizontal surface acoustic waves (SH-SAWs) are found in this theoretical report. The SH-SAW propagation is managed by the free surface of a solid when it has a direct contact with a vacuum. The studied smart solid represents the transversely isotropic piezoelectromagnetic (magnetoelectroelastic or MEE) medium that pertains to crystal symmetry class $6 \mathrm{~mm}$. In the developed theoretical treatment, the solid surface must be mechanically free. Also, the magnetic and electrical boundary conditions at the common interface between a vacuum and the solid surface read: the magnetic and electrical displacements must continue and the same for the magnetic and electrical potentials. To obtain these two new SH-SAW solutions, the natural coupling mechanisms such as $e \mu-h \alpha$ and $\varepsilon \mu-\alpha^{2}$ present in the coefficient of the magnetoelectromechanical coupling (CMEMC) can be exploited. Based on the obtained theoretical results, it is possible that a set of technical devices (filters, sensors, delay lines, lab-on-a-chip, etc.) based on smart MEE media can be developed. It is also blatant that the obtained theoretical results can be helpful for the further theoretical and experimental studies on the propagation of the plate SHwaves and the interfacial SH-waves in the MEE (composite) media. The most important issue can be the influence of the magnetoelectric effect on the SH-wave propagation. One must also be familiar with the fact that the surface, interfacial, and plate SH-waves can frequently represent a common tool for nondestructive testing and evaluation of surfaces, interfaces, and plates, respectively.

\section{Keywords}

Piezoelectromagnetic Medium, Magnetoelectric Effect, New Shear-Horizontally Polarized SAWs 


\section{Introductory Part}

There is single review work [1] concerning the piezoelectromagnetic shear-horizontal surface acoustic waves (SH-SAWs). Surface waves can propagate in a solid and are localized at the solid surface. The solid surface is free when there are no any external perturbations, but the contact with a vacuum above the surface. This report has an interest in an extra study of a smart solid (composite) material such as the transversely isotropic (hexagonal class $6 \mathrm{~mm}$ ) piezoelectromagnetic (PEM) continuum concerning any possibility of the surface wave propagation managed by the free surface.

The PEM continua are also called the magnetoelectroelastic media. They can simultaneously exhibit evidence of the following effects [2] [3]: magnetoelectric (ME), piezoelectric (PE), piezomagnetic (PM). The knowledge of PEM smart material features can serve for the creation of intelligent structures and a set of innovative technical devices to record selected environmental and internal changes. Due to the extrinsic ME effect and the coupling interaction between the electrical and magnetic fields via the elastic field of the ME continuum [3] [4], the electrical polarization can occur upon the application of a magnetic field (direct ME effect) or the magnetization can occur upon the application of an electric field (converse ME effect).

There is much review work cited in [3]-[45] on the ME effect, ME materials, and their applications because two-phase piezoelectric-piezomagnetic multiferroic composite materials with strong coupling between ferroelectric and ferromagnetic phases are frequently found [5] [6] and exhaustively studied [14]. The PEM SH-SAWs can be quite suitable for analyzing high-frequency technical devices and readily generated with the following noncontact technique [46]-[48]: electromagnetic acoustic transducers (EMATs). The use of this noncontact technique can be preferable compared with the other traditional technique that uses the PE transduction [47] [48].

Two-phase PEM composite media possessing the PE and PM phases can be exploited in different technical devices because the ME coupling in such composites represents a product property resulting from the mechani$\mathrm{cal}$ interaction between the mentioned phases. Experimental studies of the ME effect in the two-phase composites were begun in the 1970 s with pioneer works [49]-[52] to synthesize the $\mathrm{BaTiO}_{3}-\mathrm{CoFe}_{2} \mathrm{O}_{4}$ composites by a unidirectional solidification process. As a result, it was found that the obtained composites possessing the PE phase $\mathrm{BaTiO}_{3}$ and $\mathrm{PM}$ phase $\mathrm{CoFe}_{2} \mathrm{O}_{4}$ can have two orders larger value of the ME coefficient than that of the pioneer single-phase $\mathrm{ME} \mathrm{Cr}_{2} \mathrm{O}_{3}$ crystal. References [53] [54] provide the material characteristics of different $\mathrm{BaTiO}_{3}-\mathrm{CoFe}_{2} \mathrm{O}_{4}$ hexagonal $(6 \mathrm{~mm})$ composites pertaining to the (0-3) connectivity when the PE phase serving as the 3-D matrix contains the PM phase as 0-D inclusions. The reverse connectivity is also possible. Also, PEM composites can have the (2-2) connectivity. In this case, they represent a multi-layered (sandwich-like) structure composed of linear homogeneous PE and PM layers with a perfect bonding at each interface. The study of such PEM laminated (composite) structures is up to date [55].

The PEM systems can demonstrate significant interactions between the elastic, magnetic, and electric fields. This allows direct applications in sensing and actuating devices. The material parameters of the frequently used $\mathrm{BaTiO}_{3}-\mathrm{CoFe}_{2} \mathrm{O}_{4}$ and PZT-5H-Terfenol-D laminated composites can be found in [56]-[59]. It is well-known that the ME effect in the single-phase PEMs (for instance, $\mathrm{Cr}_{2} \mathrm{O}_{3}, \mathrm{LiCoPO}_{4}$, and $\mathrm{TbPO}_{4}$ [3]) is usually very small. In addition, none of the single-phase $\mathrm{ME}$ materials can have combined large and robust magnetic and electric polarizations at room temperature. However, it is a pleasure to state that the $\mathrm{Sr}_{3} \mathrm{Co}_{2} \mathrm{Fe}_{24} \mathrm{O}_{41} \mathrm{Z}$-type hexaferrite [5] was discovered in 2010. It actually possesses the realizable ME effect apt for practical uses.

It is thought that the first theoretical work on the propagation of the PEM SH-SAWs managed by the free surface was written by Melkumyan [60]. He has discovered several new SH-waves corresponding to different boundary conditions. Theoretical works [60] [61] are relevant to the studies of the SH-SAWs directed by the free surface of the hexagonal PEMs of symmetry class $6 \mathrm{~mm}$ and book [62] studies the PEM SH-SAW existence in cubic crystals. The theoretical work presented in this paper belongs to the existence of extra new SH-SAWs along the free surface of the aforementioned hexagonal medium. This paper has the purpose to discover some extra solutions (new SH-SAWs) for a set of the magnetic and electrical conditions applied at the vacuum-solid interface when the solid surface is the mechanically free. Continuity of both the electrical displacement and magnetic flux and continuity of both the magnetic and electrical potentials are treated here at the interface. Various boundary conditions for the case when a medium simultaneously possesses both the PE and PM properties are perfectly described in paper [63].

The reader can find the comprehensive theory of SH-wave propagation in the transversely isotropic media in books [61] [64] [65], of which books [64] [65] study the interfacial and plate anti-plane polarized waves, respec- 
tively. It is necessary to briefly review the theory developed in book [61] for the SH-SAW propagation along the free surface of the hexagonal $(6 \mathrm{~mm})$ PEMs. For a PEM medium, acoustic wave propagation coupled with both the electrical and magnetic potentials requires suitable thermodynamic functions and thermodynamic variables. The mechanical stress, electrical induction $(\boldsymbol{D})$, and magnetic flux $(\boldsymbol{B})$ can be chosen as the appropriate thermodynamic functions [61] in the case of linear elasticity. As a result, the thermodynamic variables are the mechanical strain, electrical field $(\boldsymbol{E})$, and magnetic field $(\boldsymbol{H})$. In such thermodynamic treatment, all the material constants can be thermodynamically determined. The electrical field components $\left(E_{i}\right)$ and the magnetic field components $\left(H_{i}\right)$ can be defined by the electrical potential $\varphi$ and magnetic potential $\psi$, respectively: $E_{i}=-\partial \varphi / \partial x_{i}$ and $H_{i}=-\partial \psi / \partial x_{i}$, where $x_{i}$ represent the real space components and the index $i$ runs from 1 to 3 .

Exploitation of the equilibrium equations and the corresponding Maxwell equations written in the form of the quasi-static approximation [66] [67] can constitute the coupled equations of motion representing partial second derivatives. Using the plane wave solution, the coupled equations of motion can be then written in the tensor form representing the Green-Christoffel equation [61]. The Green-Christoffel equation representing a polynomial is the main equation to study acoustic wave propagation coupled with both the electrical and magnetic potentials. To resolve this equation means to determine the eigenvalues and corresponding eigenvectors.

There are high symmetry propagation directions [67] [68] in which "pure" waves with the in-lane polarization and "pure" waves with the anti-pale polarization (shear-horizontal polarization) can exist. When the pure waves with the anti-plane polarization are coupled with both the electrical and magnetic potentials, the pure waves with the in-lane polarization represent purely mechanical waves, and vice versa. The appropriate cuts and propagation directions for materials with an assortment of symmetry classes are listed in works [67] [68]. It is central to state that each symmetry class has its own set of the material constants [69] [70].

For materials of symmetry class $6 \mathrm{~mm}$, the suitable propagation directions are mentioned in review paper [71] and review paper [1] exhibits the coordinate system that is fitting for PEMs, pure PEs, and pure PMs. It is necessary to mention that in the pure PEs (or PMs) the surface Bleustein-Gulyaev waves [72] [73] can propagate. Using the rectangular coordinate system $\left(x_{1}, x_{2}, x_{3}\right)$, it is necessary to clarify that the SH-SAW propagation direction, sixfold symmetry axis of the PEM material, and the surface normal must be managed along the $x_{1^{-}}, x_{2}$, and $x_{3}$-axes, respectively. Consequently, such propagation directions can support the coupling of the elastic SH-waves with both the electrical and magnetic potentials. For the case of the SH-wave propagation, the Green-Christoffel equation is simplified and all the apt eigenvalues and the corresponding eigenvectors in the form of $\left(U_{2}^{0}, U_{4}^{0}=\varphi^{0}, U_{5}^{0}=\psi^{0}\right)$ can be analytically determined.

The following section starts with the Green-Christoffel equation for the case of the SH-wave propagation in the suitable direction when there is the coupling of the SH-waves with both the magnetic and electrical potentials. It also develops the theory leading to extra two solutions of new SH-SAWs (see final formulae (60) and (66) corresponding to the coupling mechanism such as $e \mu-h \alpha$ [74]) for the certain set of the boundary conditions mentioned above. It is also worth noting that the eighth and ninth new SH-SAWs corresponding to the other coupling mechanism such as $e \alpha-h \varepsilon$ [74] were discovered in theoretical work [75]. Consequently, one has to look through the further analysis to be familiar with the explicit forms of the discovered tenth and eleventh new SH-SAWs. These extra two new solutions are possible because only one of three suitable eigenvalues depends on the phase velocity and all the apt corresponding eigenvector components do not depend on the velocity that will be demonstrated in the following section.

\section{Theory Leading to Two New Results}

In the appropriate propagation directions mentioned in the previous section, the $\mathrm{SH}$-wave propagation can be coupled with both the magnetic and electrical potentials. In the case of surface wave, the anti-plane polarized $\mathrm{SH}$-waves can be localized at the free surface of the transversely isotropic PEM continuum of symmetry class 6 $\mathrm{mm}$. The treated SH-waves propagates along the $x_{1}$-axis of the rectangular coordinate system $\left(x_{1}, x_{2}, x_{3}\right)$. In this case there is only the single mechanical displacement component $U=U_{2}$ directed along the $x_{2}$-axis. These propagation directions allow the existence of the following independent nonzero material constants: the elastic stiffness constant $C$, piezomagnetic coefficient $h$, piezoelectric constant $e$, dielectric permittivity coefficient $\varepsilon$, magnetic permeability coefficient $\mu$, and electromagnetic constant $\alpha$. They are defined by the following equalities: $C=C_{44}=C_{66}, e=e_{16}=e_{34}, h=h_{16}=h_{34}, \varepsilon=\varepsilon_{11}=\varepsilon_{33}, \mu=\mu_{11}=\mu_{33}$, and $\alpha=\alpha_{11}=\alpha_{33}$ [61].

The propagation direction can be defined by the directional cosines $\left(n_{1}, n_{2}, n_{3}\right)$ respectively directed along the 
$\left(x_{1}, x_{2}, x_{3}\right)$ axes, where $n_{1}=1, n_{2}=0$, and $n_{3} \equiv n_{3}$. They are coupled with the components $\left(k_{1}, k_{2}, k_{3}\right)$ of the wavevector $\boldsymbol{K}$ as follows: $\left(k_{1}, k_{2}, k_{3}\right)=k\left(n_{1}, n_{2}, n_{3}\right)$, where $k$ is the wavenumber in the propagation direction. All the suitable eigenvalues $n_{3}$ and the corresponding eigenvectors $\left(U^{0}, \varphi^{0}, \psi^{0}\right)=\left(U_{2}^{0}, U_{4}^{0}, U_{5}^{0}\right)$ must be disclosed. When only the SH-wave propagation is coupled with both the electrical potential $\varphi$ and the magnetic potential $\psi$, the corresponding coupled equations of motion representing the partial differential equations of the second order are written and they have the plane wave form solutions [61]. Substituting the solutions into the differential form for the coupled equations of motion, the tensor form of the coupled equations of motion can be expressed by three homogeneous equations. The differential and tensor forms of the coupled equations of motion are identical and therefore, it is possible to start the analysis with the tensor form known as the modified Green-Christoffel equation. Using the corresponding nonzero Green-Christoffel tensor components [61], the following three homogeneous equations naturally written in the matrix form can be composed:

$$
\left(\begin{array}{ccc}
C\left[m-\left(V_{p h} / V_{t 4}\right)^{2}\right] & e m & h m \\
e m & -\varepsilon m & -\alpha m \\
h m & -\alpha m & -\mu m
\end{array}\right)\left(\begin{array}{c}
U^{0} \\
\varphi^{0} \\
\psi^{0}
\end{array}\right)=\left(\begin{array}{l}
0 \\
0 \\
0
\end{array}\right)
$$

where $m=1+n_{3}^{2} ; \rho$ and $V_{p h}$ are the PEM mass density and the phase velocity, respectively. The velocity $V_{p h}$ is defined by $V_{p h}=\omega / k$, where $\omega$ is the angular frequency. Also, $V_{t 4}$ stands for the speed of the shear-horizontal bulk acoustic wave (SH-BAW). This velocity is uncoupled with both the electrical and magnetic potentials. This speed of the purely mechanical SH-wave is defined by

$$
V_{t 4}=\sqrt{C / \rho}
$$

All the appropriate eigenvalues $n_{3}$ can be determined when the determinant of the coefficient matrix in Equations (1) vanishes. Expanding the matrix determinant, the following secular equation consisting of three factors can be readily obtained:

$$
m \times m \times\left[\left(1+K_{e m}^{2}\right) m-\left(V_{p h} / V_{t 4}\right)^{2}\right]=0
$$

In expression (3), $K_{e m}^{2}$ stands for the coefficient of the magnetoelectromechanical coupling (CMEMC) defined by the following formula:

$$
K_{e m}^{2}=\frac{\mu e^{2}+\varepsilon h^{2}-2 \alpha e h}{C\left(\varepsilon \mu-\alpha^{2}\right)}=\frac{e(e \mu-h \alpha)-h(e \alpha-h \varepsilon)}{C\left(\varepsilon \mu-\alpha^{2}\right)}
$$

It is obvious that CMEMC (4) can be represented as the material parameter depending on the following three different coupling mechanisms [74] that naturally contain the electromagnetic constant $\alpha$ :

$$
\begin{gathered}
e \mu-h \alpha \\
e \alpha-h \varepsilon \\
\varepsilon \mu-\alpha^{2}
\end{gathered}
$$

It is apparent that equality (3) is satisfied as soon as one of three factors on the left-hand side is equal to zero. Thus, the first and second factors can become equal to zero for the following identical eigenvalues [61] [64] [75]:

$$
n_{3}^{(1)}=n_{3}^{(3)}=-\mathrm{j}
$$

The third factor in equation (3) provides the following suitable eigenvalue [61] [64] [75]:

$$
n_{3}^{(5)}=-\mathrm{j} \sqrt{1-\left(V_{p h} / V_{\text {tem }}\right)^{2}}
$$

where the velocity $V_{\text {tem }}$ represents the speed of the SH-BAW coupled with both the electrical and magnetic potentials and is defined as follows: 


$$
V_{\text {tem }}=V_{t 4}\left(1+K_{e m}^{2}\right)^{1 / 2}
$$

Found suitable eigenvalues (8) and (9) possess negative imaginary parts to have wave damping towards depth of the PEM material. Using them in equations (1), it is possible to reveal the corresponding eigenvectors [61]. It is natural to use the first equation in matrix form (1) to demonstrate the dependence of the eigenvector component $U^{0}$ on both the components $\varphi^{0}$ and $\psi^{0}$. Using this $U^{0}$ for the second and third equations, it is possible to get two homogeneous equations. Therefore, one can write

$$
\begin{aligned}
& U^{0}=-\frac{e m}{A} \varphi^{0}-\frac{h m}{A} \psi^{0} \\
& \left(\frac{m e^{2}}{A}+\varepsilon\right) \varphi^{0}+\left(\frac{m e h}{A}+\alpha\right) \psi^{0}=0 \\
& \left(\frac{m e h}{A}+\alpha\right) \varphi^{0}+\left(\frac{m h^{2}}{A}+\mu\right) \psi^{0}=0 \\
& A=C\left[m-\left(V_{p h} / V_{t 4}\right)^{2}\right]
\end{aligned}
$$

It is natural to utilize Equations (11) and (13) to obtain the eigenvector components such as $U^{0}, \varphi^{0}$, and $\psi^{0}$. Using $m=1+n_{3}^{2}=0$ for eigenvalues (8), it is possible to have the following eigenvector components [61] discussed in [76]:

$$
\left(U^{0(1)}, \varphi^{0(1)}, \psi^{0(1)}\right)=\left(U^{0(3)}, \varphi^{0(3)}, \psi^{0(3)}\right)=(0, \mu,-\alpha)
$$

For eigenvalue (9) with $m \neq 0$, the corresponding eigenvector components are as follows:

$$
\begin{aligned}
& \left(U^{0(5)}, \varphi^{0(5)}, \psi^{0(5)}\right)=\left(\frac{e \mu-h \alpha}{C K_{e m}^{2}},-\frac{h^{2}}{C K_{e m}^{2}}+\mu, \frac{e h}{C K_{e m}^{2}}-\alpha\right) \\
& =\frac{1}{K_{e m}^{2}}\left((e \mu-h \alpha) / C, \mu\left(K_{e m}^{2}-K_{m}^{2}\right),-\alpha\left(K_{e m}^{2}-K_{\alpha}^{2}\right)\right) .
\end{aligned}
$$

where

$$
e \varphi^{0(1)}+h \psi^{0(1)}=e \varphi^{0(5)}+h \psi^{0(5)}=e \mu-h \alpha .
$$

In expression (16), the nondimensional parameters $K_{\alpha}^{2}$ and $K_{m}^{2}$ are defined by

$$
\begin{aligned}
K_{\alpha}^{2} & =\frac{e h}{C \alpha}=\frac{\alpha e h}{C \alpha^{2}} \\
K_{m}^{2} & =\frac{h^{2}}{C \mu}
\end{aligned}
$$

where the last is called the coefficient of the magnetomechanical coupling (CMMC).

The obtained eigenvalues and the corresponding eigenvectors are employed to compose the complete mechanical displacement $U^{\Sigma}$, complete electrical potential $\varphi^{\Sigma}$, and complete magnetic potential $\psi^{\Sigma}$. Using the weight factors $F^{(1)}, F^{(3)}$, and $F^{(5)}$, these parameters are also written in the plane wave forms as follows:

$$
\begin{aligned}
U^{\Sigma} & =\sum_{p=1,3,5} F^{(p)} U^{0(p)} \exp \left[\mathrm{j} k\left(n_{1} x_{1}+n_{3}^{(p)} x_{3}-V_{p h} t\right)\right] \\
\varphi^{\Sigma} & =\sum_{p=1,3,5} F^{(p)} \varphi^{0(p)} \exp \left[\mathrm{j} k\left(n_{1} x_{1}+n_{3}^{(p)} x_{3}-V_{p h} t\right)\right] \\
\psi^{\Sigma} & =\sum_{p=1,3,5} F^{(p)} \psi^{0(p)} \exp \left[\mathrm{j} k\left(n_{1} x_{1}+n_{3}^{(p)} x_{3}-V_{p h} t\right)\right]
\end{aligned}
$$


where $t$ is time and $\mathrm{j}$ is the imaginary unity, $\mathrm{j}=(-1)^{1 / 2}$.

These weight factors can be found when the boundary conditions are applied. The mechanical, electrical, and magnetic boundary conditions are perfectly described in theoretical work [63]. For the further analysis, let's use $F_{1}, F_{2}$, and $F_{3}$ instead of $F^{(1)}, F^{(3)}$, and $F^{(5)}$, respectively. It is worth mentioning that in this case there are two identical eigenvalues (8). This fact allows the utilization of $F=F_{1}+F_{2}$ that will be naturally used below and plays a crucial role in the discovery of new SH-SAWs. Therefore, it is possible to state that this three-partial wave with $F_{1}, F_{2}$, and $F_{3}$ looks like a hidden two-partial wave with $F$ and $F_{3}$.

At the interface between the PEM medium and a vacuum $\left(x_{3}=0\right)$ the mechanically free surface requires that the normal component of the stress tensor must vanish, namely $\sigma_{32}=0$ [61]. The electrical boundary conditions at the interface include the continuity of both the electrical induction $\left(D_{3}=D^{f}\right)$ and the electrical potential $(\varphi=\varphi$ $f$ ) where the superscript $f$ relates to a vacuum. Besides, the magnetic boundary conditions at the interface include the continuity of both the magnetic flux $\left(B_{3}=B^{f}\right)$ and the magnetic potential $\left(\psi=\psi^{f}\right)$.

For the PEM medium, the parameters corresponding to the mechanical, electrical, and magnetic boundary conditions can be expressed as follows:

$$
\begin{aligned}
\sigma_{32}= & F_{1}\left[C k_{3}^{(1)} U^{0(1)}+e k_{3}^{(1)} \varphi^{0(1)}+h k_{3}^{(1)} \psi^{0(1)}\right] \\
& +F_{2}\left[C k_{3}^{(3)} U^{0(3)}+e k_{3}^{(3)} \varphi^{0(3)}+h k_{3}^{(3)} \psi^{0(3)}\right] \\
& +F_{3}\left[C k_{3}^{(5)} U^{0(5)}+e k_{3}^{(5)} \varphi^{0(5)}+h k_{3}^{(5)} \psi^{0(5)}\right] . \\
D_{3}= & F_{1}\left[e k_{3}^{(1)} U^{0(1)}-\varepsilon k_{3}^{(1)} \varphi^{0(1)}-\alpha k_{3}^{(1)} \psi^{0(1)}\right] \\
& +F_{2}\left[e k_{3}^{(3)} U^{0(3)}-\varepsilon k_{3}^{(3)} \varphi^{0(3)}-\alpha k_{3}^{(3)} \psi^{0(3)}\right] \\
& +F_{3}\left[e k_{3}^{(5)} U^{0(5)}-\varepsilon k_{3}^{(5)} \varphi^{0(5)}-\alpha k_{3}^{(5)} \psi^{0(5)}\right] . \\
\varphi= & F_{1} \varphi^{0(1)}+F_{2} \varphi^{0(3)}+F \varphi^{0(5)} \\
B_{3}= & F_{1}\left[h k_{3}^{(1)} U^{0(1)}-\alpha k_{3}^{(1)} \phi^{0(1)}-\mu k_{3}^{(1)} \psi^{0(1)}\right] \\
& +F_{2}\left[h k_{3}^{(3)} U^{0(3)}-\alpha k_{3}^{(3)} \phi^{0(3)}-\mu k_{3}^{(3)} \psi^{0(3)}\right] \\
& +F_{3}\left[h k_{3}^{(5)} U^{0(5)}-\alpha k_{3}^{(5)} \phi^{0(5)}-\mu k_{3}^{(5)} \psi^{0(5)}\right] . \\
\psi= & F_{1} \psi^{0(1)}+F_{2} \psi^{0(3)}+F_{3} \psi^{0(5)}
\end{aligned}
$$

where $F_{1}=F^{(1)}, F_{2}=F^{(3)}$, and $F_{3}=F^{(5)}$.

The corresponding vacuum parameters read:

$$
\begin{aligned}
& D^{f}=-F_{E} \varphi_{0}^{f} \mathrm{j} k_{1} \varepsilon_{0} \\
& \varphi^{f}=F_{E} \varphi_{0}^{f} \\
& B^{f}=-F_{M} \psi_{0}^{f} \mathrm{j} k_{1} \mu_{0} \\
& \psi^{f}=F_{M} \psi_{0}^{f}
\end{aligned}
$$

where $F_{E}$ and $F_{M}$ are the electrical and magnetic weight factors, respectively.

In order to elucidate the vacuum material parameters, it is essential to state that the elastic constant $C_{0}$ of the free space (vacuum) is as high as $C_{0}=0.001 \mathrm{~Pa}$ [77]. It is clearly seen that it must be multiplied by a factor of $10^{13}$ in order to be comparable with the corresponding material parameter of a solid. Thus, the neglect of this vacuum parameter is understandable in the calculations. However, the other two material parameters must be accounted. They are the magnetic permeability constant, $\mu_{0}=4 \pi \times 10^{-7}[\mathrm{H} / \mathrm{m}]=1.25663706144 \times 10^{-6}\left[\mathrm{~N} / \mathrm{A}^{2}\right]$, and the dielectric permittivity constant, $\varepsilon_{0}=10^{-7} /\left(4 \pi C_{L}^{2}\right)=0.08854187817 \times 10^{-10}[\mathrm{~F} / \mathrm{m}]$ where $C_{L}=2.99782458 \times 10^{8}[\mathrm{~m} / \mathrm{s}]$ is the speed of light in the free space. For the magnetic and electrical potentials in 
a vacuum, it is natural to write the corresponding Laplace equations of types $\Delta \psi^{f}=0$ and $\Delta \varphi^{f}=0$, where $\Delta$ denotes the differential operator called the Laplacian that forms a vector field from a scalar one.

Utilizing the equations corresponding to the mechanical, magnetic, and electrical boundary conditions written above, the following matrix form of three homogeneous equations [61] can be inscribed:

$$
\begin{aligned}
& \left(\begin{array}{ccc}
n_{3}^{(1)}\left[C U^{0(1)}+e \varphi^{0(1)}+h \psi^{0(1)}\right] & n_{3}^{(3)}\left[C U^{0(3)}+e \varphi^{0(3)}+h \psi^{0(3)}\right] & n_{3}^{(5)}\left[C U^{0(5)}+e \varphi^{0(5)}+h \psi^{0(5)}\right] \\
e n_{3}^{(1)} U^{0(1)}-\left(\varepsilon n_{3}^{(1)}-\mathrm{j} \varepsilon_{0}\right) \varphi^{0(1)}-\alpha n_{3}^{(1)} \psi^{0(1)} & e n_{3}^{(3)} U^{0(3)}-\left(\varepsilon n_{3}^{(3)}-\mathrm{j} \varepsilon_{0}\right) \varphi^{0(3)}-\alpha n_{3}^{(3)} \psi^{0(3)} & e n_{3}^{(5)} U^{0(5)}-\left(\varepsilon n_{3}^{(5)}-\mathrm{j} \varepsilon_{0}\right) \varphi^{0(5)}-\alpha n_{3}^{(5)} \psi^{0(5)} \\
h n_{3}^{(1)} U^{0(1)}-\alpha n_{3}^{(1)} \varphi^{0(1)}-\left(\mu n_{3}^{(1)}-\mathrm{j} \mu_{0}\right) \psi^{0(1)} & h n_{3}^{(3)} U^{0(3)}-\alpha n_{3}^{(3)} \varphi^{0(3)}-\left(\mu n_{3}^{(3)}-\mathrm{j} \mu_{0}\right) \psi^{0(3)} & h n_{3}^{(5)} U^{0(5)}-\alpha n_{3}^{(5)} \varphi^{0(5)}-\left(\mu n_{3}^{(5)}-\mathrm{j} \mu_{0}\right) \psi^{0(5)}
\end{array}\right) \\
& \left(\begin{array}{l}
F_{1} \\
F_{2} \\
F_{3}
\end{array}\right)=\left(\begin{array}{l}
0 \\
0 \\
0
\end{array}\right) .
\end{aligned}
$$

where the corresponding eigenvalues $n_{3}$ are used instead of $k_{3}=k n_{3}$. The vacuum material parameters $\varepsilon_{0}$ and $\mu_{0}$ are already accounted in equations (32) because the vacuum weight factors $F_{E}$ and $F_{M}$ can be naturally excluded; see the boundary conditions written above.

It is natural to use expressions (15) and (16) to simplify equations (32). As a result, the following equalities can be used to significantly simplify equations (32):

$$
\begin{aligned}
& C U^{0(1)}+e \varphi^{0(1)}+h \psi^{0(1)}=C U^{0(3)}+e \varphi^{0(3)}+h \psi^{0(3)}=e \mu-h \alpha \\
& C U^{0(5)}+e \varphi^{0(5)}+h \psi^{0(5)}=(e \mu-h \alpha) \frac{1+K_{e m}^{2}}{K_{e m}^{2}} \\
& e U^{0(1)}-\varepsilon \varphi^{0(1)}-\alpha \psi^{0(1)}=e U^{0(3)}-\varepsilon \varphi^{0(3)}-\alpha \psi^{0(3)}=-\varepsilon \mu+\alpha^{2} \\
& e U^{0(5)}-\varepsilon \varphi^{0(5)}-\alpha \psi^{0(5)}=\frac{\mu e^{2}-\alpha e h}{C K_{e m}^{2}}+\frac{\varepsilon h^{2}}{C K_{e m}^{2}}-\varepsilon \mu-\frac{\alpha e h}{C K_{e m}^{2}}+\alpha^{2}=0 \\
& h U^{0(1)}-\alpha \varphi^{0(1)}-\mu \psi^{0(1)}=h U^{0(3)}-\alpha \varphi^{0(3)}-\mu \psi^{0(3)}=-\alpha \mu+\mu \alpha=0 \\
& h U^{0(5)}-\alpha \varphi^{0(5)}-\mu \psi^{0(5)}=\frac{e \mu h-\alpha h^{2}}{C K_{e m}^{2}}+\frac{\alpha h^{2}}{C K_{e m}^{2}}-\alpha \mu-\frac{e \mu h}{C K_{e m}^{2}}+\alpha \mu=0
\end{aligned}
$$

The exploitation of equalities from (33) to (38) and eigenvalues (8) and (9) for the matrix form (32) allows one to rewrite three homogeneous equations (32) in the following simplified forms:

$$
\begin{aligned}
& (e \mu-h \alpha)\left[F_{1}+F_{2}+F_{3} \frac{1+K_{e m}^{2}}{K_{e m}^{2}} \sqrt{1-\left(\frac{V_{p h}}{V_{t e m}}\right)^{2}}\right]=0 \\
& \left(F_{1}+F_{2}\right)\left[\left(\varepsilon+\varepsilon_{0}\right) \mu-\alpha^{2}\right]+F_{3} \varepsilon_{0} \mu \frac{K_{e m}^{2}-K_{m}^{2}}{K_{e m}^{2}}=0 \\
& -\alpha\left[F_{1}+F_{2}+F_{3} \frac{K_{e m}^{2}-K_{\alpha}^{2}}{K_{e m}^{2}}\right]=0
\end{aligned}
$$

It is apparent that three homogeneous equations written in matrix form (32) with their simplified forms obtained in equations from (39) to (41) are identical. Therefore, it is possible to compose the determinant of the coefficient matrix called the determinant of the boundary conditions that must vanish to obtain a certain phase velocity $V_{p h}$ satisfying the boundary conditions. Analyzing equations from (39) to (41), it is possible to reveal that the composed determinant will have two identical columns such as the first and second ones. This peculiarity allows the conclusion such that the matrix determinant will be equal to zero at any value of the phase velocity $V_{p h}$. This means that there is uncertainty for the velocity $V_{p h}$. However, the value of the suitable SH-SAW speed must not exceed the value of the SH-BAW speed $V_{\text {tem }}$. Indeed, all the apt SH-SAW speeds satisfying the 
boundary conditions must be disclosed.

To avoid this uncertainty for the phase velocity $V_{p h}$, it is natural to use $F=F_{1}+F_{2}$ and to rewrite equations from (39) to (41) as follows:

$$
\begin{aligned}
& (e \mu-h \alpha)\left[F+F_{3} \frac{1+K_{e m}^{2}}{K_{e m}^{2}} \sqrt{1-\left(\frac{V_{p h}}{V_{t e m}}\right)^{2}}\right]=0 \\
& F\left[\left(\varepsilon+\varepsilon_{0}\right) \mu-\alpha^{2}\right]+F_{3} \varepsilon_{0} \mu \frac{K_{e m}^{2}-K_{m}^{2}}{K_{e m}^{2}}=0 \\
& -\alpha\left[F+F_{3} \frac{K_{e m}^{2}-K_{\alpha}^{2}}{K_{e m}^{2}}\right]=0
\end{aligned}
$$

It is visible that the set of equations from (42) to (44) is the same to the set of equations from (39) to (41) because $F=F_{1}+F_{2}$ was used. There is already no uncertainty of the phase velocity $V_{p h}$ for the set of equations from (42) to (44). This is true because they represent three homogeneous equations in two unknown weight factors such as $F$ and $F_{3}$. It is well-known that such new system of equations can have a certain solution of the velocity $V_{p h}$ when three equations from (42) to (44) are dependable from each other. This happens as soon as one equation represents a sum of two others. Therefore, three equations from (42) to (44) must be properly transformed into a set of suitable consistent equations.

In order to obtain three consistent equations, it is natural to treat equation (43) as the main one and multiply Equations (42) and (44) by $\left(\varepsilon+\varepsilon_{0}\right) \mu /(e \mu-h \alpha)$ and $\alpha$, respectively. So, one can get the following three homogeneous equations that are already consistent and have the same dimension:

$$
\begin{aligned}
& \left(\varepsilon+\varepsilon_{0}\right) \mu\left[F+F_{3} \frac{1+K_{e m}^{2}}{K_{e m}^{2}} \sqrt{1-\left(\frac{V_{p h}}{V_{t e m}}\right)^{2}}\right]=0 \\
& F\left[\left(\varepsilon+\varepsilon_{0}\right) \mu-\alpha^{2}\right]+F_{3} \varepsilon_{0} \mu \frac{K_{e m}^{2}-K_{m}^{2}}{K_{e m}^{2}}=0 \\
& -\alpha^{2}\left[F+F_{3} \frac{K_{e m}^{2}-K_{\alpha}^{2}}{K_{e m}^{2}}\right]=0
\end{aligned}
$$

So, three equations from (45) to (47) in two unknowns $F$ and $F_{3}$ are consistent because the left-hand side of main equation (46) can become equal to zero as soon as equations (45) and (47) are successively subtracted from equation (46). This subtraction leads to the certain velocity of new SH-SAW. Also, main equation (46) can be used to disclose the explicit forms of the weight factors $F$ and $F_{3}$. It is also convenient to rewrite three equations from (45) to (47) as the following set of two equations in two unknowns $F$ and $F_{3}$ :

$$
\begin{aligned}
& F\left[\left(\varepsilon+\varepsilon_{0}\right) \mu-\alpha^{2}\right]+F_{3}\left[\alpha^{2} \frac{K_{e m}^{2}-K_{\alpha}^{2}}{K_{e m}^{2}}+\left(\varepsilon+\varepsilon_{0}\right) \mu \frac{1+K_{e m}^{2}}{K_{e m}^{2}} \sqrt{1-\left(\frac{V_{p h}}{V_{t e m}}\right)^{2}}\right]=0 \\
& F\left[\left(\varepsilon+\varepsilon_{0}\right) \mu-\alpha^{2}\right]+F_{3} \varepsilon_{0} \mu \frac{K_{e m}^{2}-K_{m}^{2}}{K_{e m}^{2}}=0
\end{aligned}
$$

where Equation (48) represents a sum of equations (45) and (47).

It is clearly seen in the first term of equations (48) and (49) that the factor at $F$ such as $\left[\left(\varepsilon+\varepsilon_{0}\right) \mu-\alpha^{2}\right]$ can be interpreted as coupling mechanism (7) of CMEMC (4) such as $\left(\varepsilon \mu-\alpha^{2}\right)$ when the vacuum electrical parameter $\varepsilon_{0}$ must be also included. To determine the SH-SAW velocity, it is necessary to subtract equation (48) from Equation (49), or vice versa, because they must be identical. So, the value of the velocity $V_{\text {new2 }}$ of the new SH-SAW recently discovered in book [61] (the new SH-SAW velocity is defined by expression (120) in the book, see also papers [1] [78]) can be calculated with the following explicit formula: 


$$
\begin{aligned}
V_{\text {new } 2} & =V_{\text {tem }}\left[1-\left(\frac{K_{e m}^{2}-K_{m}^{2}+\alpha^{2} C_{L}^{2} \frac{\mu_{0}}{\mu}\left(K_{e m}^{2}-K_{\alpha}^{2}\right)}{\left(1+K_{e m}^{2}\right)\left(1+\frac{\varepsilon}{\varepsilon_{0}}\right)}\right)^{2}\right]^{1 / 2} \\
& =V_{\text {tem }}\left[1-\left(\frac{(e \mu-h \alpha)\left[e\left(\varepsilon_{0} \mu+\alpha^{2}\right)-\alpha h\left(\varepsilon+\varepsilon_{0}\right)\right]}{\left(\varepsilon+\varepsilon_{0}\right) \mu\left(C \varepsilon \mu-C \alpha^{2}+\mu e^{2}+\varepsilon h^{2}-2 \alpha e h\right)}\right)^{2}\right]^{1 / 2}
\end{aligned}
$$

In formula (50) there are two possibilities when $V_{\text {new } 2}=V_{\text {tem }}$ occurs: $e \mu-h \alpha=0$ and $e\left(\varepsilon_{0} \mu+\alpha^{2}\right)-\alpha h\left(\varepsilon+\varepsilon_{0}\right)=0$. Also, the speed of light $C_{L}$ in a vacuum is defined by

$$
C_{L}^{2}=\frac{1}{\varepsilon_{0} \mu_{0}}
$$

It is worth mentioning that the SH-SAW defined by expression (50) represents one of the seven new SHSAWs recently discovered in book [61]. This new SH-SAW can propagate along the free surface of the hexagonal PEM medium of symmetry class $6 \mathrm{~mm}$. Also, it is natural to demonstrate the case when the piezomagnetic and electromagnetic constants vanish, namely $h=0$ and $\alpha=0$. In this case, the PEM SH-SAW defined by expression (50) reduces to the well-known velocity $V_{B G p e}$ of the surface Bleustein-Gulyaev waves [72] [73] propagating in a purely piezoelectric solid. The velocity $V_{B G p e}$ is defined by

$$
V_{B G p e}=V_{t e}\left[1-\left(\frac{K_{e}^{2}}{\left(1+K_{e}^{2}\right)\left(1+\varepsilon / \varepsilon_{0}\right)}\right)^{2}\right]^{1 / 2}
$$

In definition (52), $V_{t e}$ and $K_{e}^{2}$ respectively stand for the SH-BAW velocity coupled with the electrical potential and the coefficient of the electromechanical coupling (CEMC). They represent very important material characteristics of a pure piezoelectrics and read as follows:

$$
\begin{aligned}
& V_{t e}=V_{t 4}\left(1+K_{e}^{2}\right)^{1 / 2} \\
& K_{e}^{2}=\frac{e^{2}}{C \varepsilon}
\end{aligned}
$$

In formula (53), the velocity $V_{t 4}$ is defined by expression (2).

Formula (50) for the new SH-SAW velocity discovered in book [61] is given in this paper for comparison with the other new results obtained below. The main purpose of this paper is to discover additional new $\mathrm{SH}$ SAWs that can propagate in the PEM using the same set of the boundary conditions at the vacuum-solid interface: $\sigma_{32}=0, \varphi=\varphi^{f}, D=D^{f}, \psi=\psi^{f}$, and $B=B^{f}$. Therefore, two new solutions for new SH-SAW propagation are obtained in subdivisions (i) and (ii) below. Also, it is necessary to state that this theoretical study can be useful for constitution of a set of technical devices based on smart PEM solids and the further researches on the propagation of the plate and interfacial $\mathrm{SH}$-waves.

(i) Similar to the theory developed above for the PEM SH-wave propagation (see also in book [61]) it is possible to start with the analysis of three homogeneous equations from (42) to (44) in two unknowns $F$ and $F_{3}$. The second possibility for coupling mechanism (7) of CMEMC (4) such as $\left(\varepsilon \mu-\alpha^{2}\right)$ can be also treated when there is also the coupling with the vacuum electrical constant $\varepsilon_{0}$. Therefore, Equation (43) with the factor at $F$ such as $\left[\varepsilon\left(\mu+\mu_{0}\right)-\alpha^{2}\right]$ is the main equation that is not changed for this case. This main equation must couple Equations (42) and (44) together forming a system of three homogeneous equations in two unknown weight factors $F$ and $F_{3}$. It is flagrant that these three equations can become dependent on each other as soon as Equation (42) is multiplied by $\left(\varepsilon \mu-\alpha^{2}\right) /(e \mu-h \alpha)$ and Equation (44) is multiplied by $-\varepsilon_{0} \mu / \alpha$. Accordingly, three equations from (42) to (44) can be rewritten as follows: 


$$
\begin{aligned}
& \left(\varepsilon \mu-\alpha^{2}\right)\left[F+F_{3} \frac{1+K_{e m}^{2}}{K_{e m}^{2}} \sqrt{1-\left(\frac{V_{p h}}{V_{t e m}}\right)^{2}}\right]=0 \\
& F\left[\left(\varepsilon+\varepsilon_{0}\right) \mu-\alpha^{2}\right]+F_{3} \varepsilon_{0} \mu \frac{K_{e m}^{2}-K_{m}^{2}}{K_{e m}^{2}}=0 \\
& \varepsilon_{0} \mu\left[F+F_{3} \frac{K_{e m}^{2}-K_{\alpha}^{2}}{K_{e m}^{2}}\right]=0
\end{aligned}
$$

It is flagrant that it is convenient to cope with the set of two homogeneous equations in two unknowns $F$ and $F_{3}$ instead of three equations from (55) to (57) written above. A sum of Equations (55) and (57) leads to the following convenient set of equations:

$$
\begin{aligned}
& F\left[\left(\varepsilon+\varepsilon_{0}\right) \mu-\alpha^{2}\right]+F_{3}\left[\varepsilon_{0} \mu \frac{K_{e m}^{2}-K_{\alpha}^{2}}{K_{e m}^{2}}+\left(\varepsilon \mu-\alpha^{2}\right) \frac{1+K_{e m}^{2}}{K_{e m}^{2}} \sqrt{1-\left(\frac{V_{p h}}{V_{t e m}}\right)^{2}}\right]=0 \\
& F\left[\left(\varepsilon+\varepsilon_{0}\right) \mu-\alpha^{2}\right]+F_{3} \varepsilon_{0} \mu \frac{K_{e m}^{2}-K_{m}^{2}}{K_{e m}^{2}}=0
\end{aligned}
$$

Therefore, the velocity $V_{\text {new10 }}$ of the tenth new anti-plane polarized SAW propagating along the free surface of the PEM medium is obtained by a subtraction of Equation (58) from Equation (59). Also, the velocity $V_{\text {new10 }}$ can be obtained by a successive subtraction of Equations (55) and (57) from main Equation (56). Thus, this reads:

$$
\begin{aligned}
V_{\text {new10 }} & =V_{\text {tem }}\left[1-\left(\frac{\varepsilon_{0} \mu}{\varepsilon \mu-\alpha^{2}} \frac{K_{m}^{2}-K_{\alpha}^{2}}{1+K_{e m}^{2}}\right)^{2}\right]^{1 / 2} \\
& =V_{\text {tem }}\left[1-\left(-\frac{h \varepsilon_{0}}{e \alpha} \frac{e(e \mu-h \alpha)}{C \varepsilon \mu-C \alpha^{2}+\mu e^{2}+\varepsilon h^{2}-2 \alpha e h}\right)^{2}\right]^{1 / 2}
\end{aligned}
$$

In expression (60), the following equality $e \mu=h \alpha$ results in $V_{\text {new10 }}=V_{\text {tem }}$.

So, it is possible to state that the new SH-SAW discovered in this paper can propagate with the velocity $V_{\text {new10 }}$ expressed by formula (60). This is the new solution that was not considered in book [61]. Concerning the SH-wave propagation in the PEM plates [65], solution (60) discovered in this paper is more preferable and convenient than solution (50) discovered in book [61]. Also, solution (60) looks like simple one compared with solution (50). However, one can find a very interesting peculiarity: $K_{\alpha}^{2}(\alpha \rightarrow 0) \rightarrow \infty$ results in the fact that the new SH-SAW defined by expression (60) cannot exist because the expression under the square root in formula (60) can have a negative sign resulting in an imaginary value of the SH-SAW velocity. This peculiarity does not exist for solution (50) allowing SH-SAW propagation for very small values of the electromagnetic constant $\alpha$, even for $\alpha=0$.

(ii) Consider the second case that also leads to discovery of an extra new SH-SAW. However, it is essential to clarify why this case must be considered. The author of this paper has understood that this case can be possible after the study of the interfacial SH-wave propagation directed by the perfectly bonded interface between two dissimilar PEMs of symmetry class $6 \mathrm{~mm}$ [64]. This case is relevant to coupling mechanism (5) of CMEMC (4) such as $(e \mu-h \alpha)$.

Let's start with the analysis of three equations from (42) to (44). It is indispensable to treat Equation (42) as the main equation that couples Equations (43) and (44) in a set of three homogeneous equations to make them consistent. For this purpose, it is necessary to multiply the left-hand side of Equation (43) by

$e \mu /\left[\varepsilon\left(\mu+\mu_{0}\right)-\alpha^{2}\right]$ and the one of Equation (44) by the piezomagnetic constant $h$ because it already has the factor such as the constant $\alpha$. As a result, three equations from (42) to (44) can be rewritten as follows:

$$
(e \mu-h \alpha)\left[F+F_{3} \frac{1+K_{e m}^{2}}{K_{e m}^{2}} \sqrt{1-\left(\frac{V_{p h}}{V_{t e m}}\right)^{2}}\right]=0
$$




$$
\begin{aligned}
& e \mu\left[F+F_{3} \frac{\varepsilon_{0} \mu}{\left(\varepsilon+\varepsilon_{0}\right) \mu-\alpha^{2}} \frac{K_{e m}^{2}-K_{m}^{2}}{K_{e m}^{2}}\right]=0 \\
& -h \alpha\left[F+F_{3} \frac{K_{e m}^{2}-K_{\alpha}^{2}}{K_{e m}^{2}}\right]=0
\end{aligned}
$$

The set of three equations from (61) to (63) can be rewritten as a set of two corresponding equations for convenience. Indeed, a sum of Equations (62) and (63) allows one to compose the following apt set of two homogeneous equations:

$$
\begin{gathered}
(e \mu-h \alpha)\left[F+F_{3} \frac{1+K_{e m}^{2}}{K_{e m}^{2}} \sqrt{1-\left(\frac{V_{p h}}{V_{t e m}}\right)^{2}}\right]=0 \\
F(e \mu-h \alpha)+F_{3}\left[-h \alpha \frac{K_{e m}^{2}-K_{\alpha}^{2}}{K_{e m}^{2}}+e \mu \frac{\varepsilon_{0} \mu}{\left(\varepsilon+\varepsilon_{0}\right) \mu-\alpha^{2}} \frac{K_{e m}^{2}-K_{m}^{2}}{K_{e m}^{2}}\right]=0
\end{gathered}
$$

These two equations lead to the following explicit form for the velocity $V_{\text {new11 }}$ of the eleventh new SH-SAW:

$$
\begin{aligned}
V_{\text {new1 } 1} & =V_{\text {tem }}\left[1-\left(-\frac{h \alpha}{e \mu-h \alpha} \frac{K_{e m}^{2}-K_{\alpha}^{2}}{1+K_{e m}^{2}}+\frac{e \mu}{e \mu-h \alpha} \frac{\varepsilon_{0} \mu}{\left(\varepsilon+\varepsilon_{0}\right) \mu-\alpha^{2}} \frac{K_{e m}^{2}-K_{m}^{2}}{1+K_{e m}^{2}}\right)^{2}\right]^{1 / 2} \\
& =V_{\text {tem }}\left[1-\left(\frac{\varepsilon_{0} \mu e(e \mu-h \alpha)-\left(\varepsilon \mu+\varepsilon_{0} \mu-\alpha^{2}\right) h(e \alpha-h \varepsilon)}{\left(\varepsilon \mu+\varepsilon_{0} \mu-\alpha^{2}\right)\left(C \varepsilon \mu-C \alpha^{2}+\mu e^{2}+\varepsilon h^{2}-2 \alpha e h\right)}\right)^{2}\right]^{1 / 2}
\end{aligned}
$$

It is obvious that the following equality $V_{\text {new11 }}=V_{\text {tem }}$ occurs in expression (66) as soon as one treats the case of $\varepsilon_{0} \mu e(e \mu-h \alpha)-\left(\varepsilon \mu+\varepsilon_{0} \mu-\alpha^{2}\right) h(e \alpha-h \varepsilon)=0$. This equation can be also rewritten as $\varepsilon_{0} C \mu K_{e m}^{2}=h(e \alpha-h \varepsilon)$.

For the case of a very small value of the constant $\alpha(\alpha \rightarrow 0)$ formula (66) reduces to the following form:

$$
V_{\text {new1 } \_0}=V_{\text {tem0 }}\left[1-\left(-\frac{K_{m}^{2}}{1+K_{e}^{2}+K_{m}^{2}}+\frac{K_{e}^{2}}{\left(1+K_{e}^{2}+K_{m}^{2}\right)\left(1+\varepsilon / \varepsilon_{0}\right)}\right)^{2}\right]^{1 / 2}
$$

where

$$
\begin{aligned}
& V_{\text {tem } 0}=V_{t 4}\left(1+K_{e}^{2}+K_{m}^{2}\right)^{1 / 2} \\
& K_{e m}^{2}(\alpha \rightarrow 0) \rightarrow K_{e}^{2}+K_{m}^{2}
\end{aligned}
$$

With $h=0$, formula (67) reduces to formula (53) for the velocity $V_{B G p e}$ of the surface Bleustein-Gulyaev wave [72] [73] existing in a purely piezoelectric solid. So, it is possible to conclude that the consideration of CMEMC coupling mechanism (5) such as $(e \mu-h \alpha)$ results in the new SH-SAW propagating with the velocity $V_{\text {new11 }}$ defined by explicit form (66). Solutions (50), (60), and (66) are true because they are based on the natural coupling mechanisms of the CMEMC.

\section{Numerical Results and Discussion}

Comparative numerical calculations are listed in Table 1 for the different $\mathrm{BaTiO}_{3}-\mathrm{CoFe}_{2} \mathrm{O}_{4}$ composites. For comparison, the table provides results of the calculations of the propagation speeds for six known SH-SAWs that can exist for the same set of the electrical and magnetic boundary conditions: the velocities $V_{\text {new1 }}, V_{\text {new2 }}$, $V_{\text {new8 }}, V_{\text {new9 }}, V_{\text {new10}}$, and $V_{\text {new11 }}$ of the first, second, eighth, ninth, tenth, eleventh new SH-SAWs. The reader can find that all the speeds of the studied new SH-waves are slower than the SH-BAW speed $V_{\text {tem }}$. The interesting issue is the existence of the eighth and tenth new SH-SAWs. The eighth can exist only for the $20 \%$ volume fraction 
Table 1. The material and wave characteristics of the piezoelectromagnetic composites consisting of $\mathrm{BaTiO}_{3}-\mathrm{CoFe}_{2} \mathrm{O}_{4}$ of class $6 \mathrm{~mm}$. Following the results given in papers [53] [54], the material constants are given as percentage volume fraction (VF) of $\mathrm{BaTiO}_{3}$ in the composites consisting of $\mathrm{BaTiO}_{3}-\mathrm{CoFe}_{2} \mathrm{O}_{4}$. It is worth noting that the magnetic permeability of a vacuum is $\mu_{0}=4 \pi \times 10^{-7}\left[\mathrm{H} \cdot \mathrm{m}^{-1}\right] \sim 12.566371 \times 10^{-7}\left[(\mathrm{~V} \cdot \mathrm{s}) \cdot(\mathrm{A} \cdot \mathrm{m})^{-1}\right] ; 10^{-12} \mathrm{~N} \cdot \mathrm{s} /(\mathrm{V} \cdot \mathrm{C})=\mathrm{ps} / \mathrm{m} ; \mathrm{F}=\mathrm{C} / \mathrm{V} ; \mathrm{T}=\mathrm{Tesla}=$ $\mathrm{N} \cdot(\mathrm{A} \cdot \mathrm{m})^{-1}$. The mass density is assumed the same $\rho=5730\left[\mathrm{~kg} / \mathrm{m}^{3}\right]$.

\begin{tabular}{ccccccc}
\hline Composite VF & $0 \%$ & $20 \%$ & $40 \%$ & $60 \%$ & $80 \%$ & $100 \%$ \\
\hline$C, 10^{10}\left[\mathrm{~N} / \mathrm{m}^{2}\right]$ & 4.53 & 4.50 & 4.50 & 4.50 & 5.00 & 4.30 \\
$e\left[\mathrm{C} / \mathrm{m}^{2}\right]$ & 0 & 0.1 & 0.2 & 0.3 & 0.4 & 11.6 \\
$h[\mathrm{~T}]$ & 560 & 340 & 220 & 180 & 80 & 0 \\
$\varepsilon, 10^{-10}[\mathrm{~F} / \mathrm{m}]$ & 0.8 & 3.3 & 8.0 & 9.0 & 10.0 & 112.0 \\
$\mu, 10^{-6}\left[\mathrm{~N} / \mathrm{A}^{2}\right]$ & -590 & -390 & -250 & -150 & -80 & 5.0 \\
$\alpha, 10^{-12}[\mathrm{~N} \cdot \mathrm{s} / \mathrm{V} \cdot \mathrm{C}]$ & 0 & 2.8 & 4.8 & 6.0 & 6.8 & 0 \\
$K_{e m}^{2}$ & - & -0.00591346104 & -0.003191064178 & -0.00257767111 & 0.001600108799 & - \\
$V_{\text {t4 }}[\mathrm{m} / \mathrm{s}]$ & 2811.71818686 & 2802.39239604 & 2802.39239604 & 2802.39239604 & 2953.98095634 & 2739.40924320 \\
$V_{\text {tem }}[\mathrm{m} / \mathrm{s}]$ & - & 2794.09419095 & 2797.91751629 & 2798.77824255 & 2956.34335715 & - \\
$V_{\text {new1 }}[\mathrm{m} / \mathrm{s}]$ & - & 2794.09419031 & 2797.91751562 & 2798.77824023 & 2956.34335618 & - \\
$V_{\text {new } 2}[\mathrm{~m} / \mathrm{s}]$ & - & 2794.09419051 & 2797.91751608 & 2798.77824189 & 2956.34335598 & - \\
$V_{\text {new8 }}[\mathrm{m} / \mathrm{s}]$ & - & 1354.51142927 & does not exist & does not exist & does not exist & - \\
$V_{\text {new9 }}[\mathrm{m} / \mathrm{s}]$ & - & 2794.09350866 & 2797.91570936 & 2798.77104004 & 2956.32802670 & - \\
$V_{\text {new10 }}[\mathrm{m} / \mathrm{s}]$ & - & does not exist & does not exist & does not exist & 1640.10493727 & - \\
$V_{\text {new11 }}[\mathrm{m} / \mathrm{s}]$ & - & 2794.03318064 & 2797.89160405 & 2798.74612598 & 2956.33971662 & - \\
\hline
\end{tabular}

of $\mathrm{BaTiO}_{3}$ in the composites consisting of $\mathrm{BaTiO}_{3}-\mathrm{CoFe}_{2} \mathrm{O}_{4}$. On the other hand, the tenth can exist only for the $80 \%$ volume fraction. This fact can be explained by a strong dependence of the nondimensional parameter $\alpha^{2} / \varepsilon \mu$. This interesting found moment by the numerical study can be researched in the future more widely because this short report theoretically predicts and numerically demonstrates the existence of the new SH-SAWs.

Let's continue our debates on the following problem: how many surface SH-waves can exist in the transversely isotropic piezoelectromagnetics. This is an important question because there is an opinion that in this particular case, an infinite number of analytical solutions obtained in explicit forms can be found. For today, the author can certainly state that as many as fourteen different SH-SAWs can propagate treating different sets of the electrical and magnetic boundary conditions. Indeed, the author of this theoretical report can agree only with three SH-SAWs of twelve theoretically discovered by Melkumyan [60]. Seven new SH-SAWs were theoretically discovered in book [61], two new SH-SAWs were discovered in paper [75], and extra two new SH-SAWs were discovered in this report. The number of possible SH-waves is big but it does not approach an infinity. The reader can check the relatively simple mathematics used above in this report to analytically find possible extra solutions.

Concerning the boundary conditions used in this report, they represent the most complicated case. Therefore, more possibilities can exist and as many as six new SH-SAWs were discovered: the first and second in book [61], the eighth and ninth in paper [75], and the tenth and eleventh in this report. The existence of six SH-SAWs for the same set of the boundary conditions at the solid-vacuum boundary $\left(\sigma_{32}=0, \varphi=\varphi^{f}, D=D^{f}, \psi=\psi^{f}\right.$, and $B$ $=B^{f}$ ) can be naturally explain by the existence of the different possible coupling mechanisms of the CMEMC such as $e \alpha-h \varepsilon, e \mu-h \alpha$, and $\varepsilon \mu-\alpha^{2}$. It is possible to assume that the first two mechanisms represent exchange ones and only the third can relate to the magnetoelectric effect. Therefore, different solutions correspond to different CMEMC coupling mechanisms. The other important factor that influence of the number of possible SH-SAWs is the fact that there are two different sets of the eigenvector components for the same eigenvalue. 
Utilization of two different sets of the eigenvector components can frequently lead to two different solutions. This fact certainly increases the number of possible SH-SAWs that can propagate in the transversely isotropic piezoelectromagnetics. It is also discussed that so many solutions for the transversely isotropic case are possible because only one of three suitable eigenvalues defined by formulae (8) and (9) depends on the phase velocity and all the apt corresponding eigenvector components do not depend on the velocity. The different picture there is for the problem of SH-SAW propagation in the cubic piezoelectromagnetics. It was numerically found in book [62] that two different sets of the eigenvector components lead to the same result for the propagation velocity. As a result, only seven possible new SH-SAWs were numerically found in [62]: two different sets of the eigenvector components actually lead here to the same result for the propagation velocity for each possible set of the electrical and magnetic boundary conditions. The interesting issue is that the solutions can be obtained only by an analytical method for the transversely isotropic case but the solutions (after expanding the determinant of the boundary conditions) can be obtained only by a numerical method for the cubic case. The author will be glad if one can find analytical solutions for the cubic case. Also, the solution for the surface Bleustein-GulyaevMelkumyan wave can be also found analytically in the cubic piezoelectromagnetics [62].

Finally, some incorrect solutions obtained in papers [79] [80] and mentioned in review [1] for the propagation problem of SH-wave managed by the PEM free surface are also exist. It is possible to discuss them in a few words. These incorrect solutions pertain to the same set of the boundary conditions at the solid-vacuum boundary: $\sigma_{32}=0, \varphi=\varphi^{f}, D=D^{f}, \psi=\psi^{f}$, and $B=B^{f}$. The authors of theoretical articles [79] [80] have used the other theoretical methods leading to the other forms [1] that are different from formulae (50), (60), and (66). Moreover, their results discussed in review [1] even differ from each other. Review paper [1] has also exhibited that their results are incorrect because they can definitely mix two different eigenvectors for the same material. The eigenvector mixing is possible when one eigenvector is used for one material and the second for the second material for the problem of the interfacial SH-wave propagation along the common interface between two dissimilar piezoelectromagnetics that was analytically demonstrated in paper [81]. Besides, the authors of papers [79] [80] did not demonstrate that they have found suitable eigenvectors. It is worth noting that to find all the suitable eigenvalues and the corresponding eigenvectors is the main mathematical procedure to resolve the coupled equations of motion. Therefore, the authors of papers [79] [80] did not find any true solutions for the coupled equations of motion and the author of this paper cannot agree with their solutions. Their method can be used for the study of this particular and particularistic case and cannot be used for the other cases, for instance, for the problem of the SH-SAW existence in the cubic piezoelectromagnetics.

\section{Conclusion}

This theoretical work has demonstrated that extra two new solutions of new SH-SAWs propagating along the free surface of the transversely isotropic PEM medium of symmetry class $6 \mathrm{~mm}$ can be found. The discovered two SH-SAWs relate to the case of $\sigma_{32}=0, \varphi=\varphi^{f}, D=D^{f}, \psi=\psi^{f}$, and $B=B^{f}$ representing the boundary conditions at the interface between the PEM and a vacuum. The found solutions correspond to two natural coupling mechanisms such as $e \mu-h \alpha$ and $\varepsilon \mu-\alpha^{2}$ in the coefficient of the magnetoelectromechanical coupling (CMEMC). Comparative numerical calculations are listed in the table for the different $\mathrm{BaTiO}_{3}-\mathrm{CoFe}_{2} \mathrm{O}_{4}$ composites. The obtained theoretical results can be useful for constitution of an assortment of technical devices based on smart PEM materials. Also, it is thought that the obtained theoretical results can be useful for development of some further research on the propagation of the interfacial SH-waves and the plate SH-waves in the PEM (composite) systems are required to better understand their properties. Different SH-waves actually represent an interest in sensor technologies, nondestructive testing and evaluation of surfaces, interfaces, and plates.

\section{Acknowledgements}

The author is thankful to Professor Dr. V.P. Zhereb from the Siberian Federal University (Krasnoyarsk, Siberia, Russia) for fruitful discussions and his advice to submit the paper to the Journal. The author is also thankful to the referees for their valuable comments and suggestions to improve the paper quality for the Journal reader.

\section{References}

[1] Zakharenko, A.A. (2013) Piezoelectromagnetic SH-SAWs: A Review. Canadian Journal of Pure \& Applied Sciences (SENRA Academic Publishers, Burnaby, British Columbia, Canada), 7, 2227-2240. 
[2] Nan, C.W. (1994) Magnetoelectric Effect in Composites of Piezoelectric and Piezomagnetic Phases. Physical Review B, 50, 6082-6088. http://dx.doi.org/10.1103/PhysRevB.50.6082

[3] Fiebig, M. (2005) Revival of the Magnetoelectric Effect. Journal of Physics D: Applied Physics, 38, R123-R152. http://dx.doi.org/10.1088/0022-3727/38/8/R01

[4] Özgür, Ü., Alivov, Ya. and Morkoç, H. (2009) Microwave Ferrites, Part 2: Passive Components and Electrical Tuning. Journal of Materials Science: Materials in Electronics, 20, 911-952. http://dx.doi.org/10.1007/s10854-009-9924-1

[5] Kimura, T. (2012) Magnetoelectric Hexaferrites. Annual Review of Condensed Matter Physics, 3, 93-110. http://dx.doi.org/10.1146/annurev-conmatphys-020911-125101

[6] Pullar, R.C. (2012) Hexagonal Ferrites: A Review of the Synthesis, Properties and Applications of Hexaferrite Ceramics. Progress in Materials Science, 57, 1191-1334. http://dx.doi.org/10.1016/j.pmatsci.2012.04.001

[7] Park, Ch.-S. and Priya, Sh. (2012) Broadband/Wideband Magnetoelectric Response.Advances in Condensed Matter Physics (Hindawi Publishing Corporation), 2012, Article ID: 323165.

[8] Bichurin, M.I., Petrov, V.M. and Petrov, R.V. (2012) Direct and Inverse Magnetoelectric Effect in Layered Composites in Electromechanical Resonance Range: A Review. Journal of Magnetism and Magnetic Materials, 324, 35483550. http://dx.doi.org/10.1016/j.jmmm.2012.02.086

[9] Chen, T., Li, S. and Sun, H. (2012) Metamaterials Application in Sensing. MDPI Sensors, 12, 2742-2765. http://dx.doi.org/10.3390/s120302742

[10] Bichurin, M., Petrov, V., Zakharov, A., Kovalenko, D., Yang, S.Ch., Maurya, D., Bedekar, V. and Priya, Sh. (2011) Magnetoelectric Interactions in Lead-Based and Lead-Free Composites. Materials, 4, 651-702. http://dx.doi.org/10.3390/ma4040651

[11] Srinivasan, G. (2010) Magnetoelectric Composites. Annual Review of Materials Research, 40, 153-178. http://dx.doi.org/10.1146/annurev-matsci-070909-104459

[12] Zhai, J., Xing, Z.-P., Dong, S.-X., Li, J.-F. and Viehland, D. (2008) Magnetoelectric Laminate Composites: An Overview. Journal of the American Ceramic Society, 91, 351-358. http://dx.doi.org/10.1111/j.1551-2916.2008.02259.x

[13] Nan, C.W., Bichurin, M.I., Dong, S.X., Viehland, D. and Srinivasan, G. (2008) Multiferroic Magnetoelectric Composites: Historical Perspective, Status, and Future Directions. Journal of Applied Physics, 103, Article ID: 031101. http://dx.doi.org/10.1063/1.2836410

[14] Eerenstein, W., Mathur, N.D. and Scott, J.F. (2006) Multiferroic and Magnetoelectric Materials. Nature, 442, $759-765$. http://dx.doi.org/10.1038/nature05023

[15] Spaldin, N.A. and Fiebig, M. (2005) The Renaissance of Magnetoelectric Multiferroics. Science, 309, 391-392. http://dx.doi.org/10.1126/science.1113357

[16] Fiebig, M., Pavlov, V.V. and Pisarev, R.V. (2005) Magnetoelectric Phase Control in Multiferroic Manganites. Journal of the Optical Society of America B, 22, 96-118. http://dx.doi.org/10.1364/JOSAB.22.000096

[17] Khomskii, D.I. (2006) Multiferroics: Different Ways to Combine Magnetism and Ferroelectricity. Journal of Magnetism and Magnetic Materials, 306, 1-8. http://dx.doi.org/10.1016/j.jmmm.2006.01.238

[18] Cheong, S.-W. and Mostovoy, M. (2007) Multiferroics: A Magnetic Twist for Ferroelectricity. Nature Materials, 6, 13-20. http://dx.doi.org/10.1038/nmat1804

[19] Ramesh, R. and Spaldin, N.A. (2007) Multiferroics: Progress and Prospects in Thin Films. Nature Materials, 6, 21-29. http://dx.doi.org/10.1038/nmat1805

[20] Kimura, T. (2007) Spiral Magnets as Magnetoelectrics. Annual Review of Materials Research, 37, 387-413. http://dx.doi.org/10.1146/annurev.matsci.37.052506.084259

[21] Kimura, T., Goto, T., Shintani, H., Ishizaka, K., Arima, T. and Tokura, Y. (2003) Magnetic Control of Ferroelectric Polarization. Nature, 426, 55-58. http://dx.doi.org/10.1038/nature02018

[22] Wang, K.F., Liu, J.-M. and Ren, Z.F. (2009) Multiferroicity: The Coupling between Magnetic and Polarization Orders. Advances in Physics, 58, 321-448. http://dx.doi.org/10.1080/00018730902920554

[23] Ramesh, R. (2009) Materials Science: Emerging Routes to Multiferroics. Nature, 461, 1218-1219. http://dx.doi.org/10.1038/4611218a

[24] Delaney, K.T., Mostovoy, M. and Spaldin, N.A. (2009) Superexchange-Driven Magnetoelectricity in Magnetic Vertices. Physical Review Letters, 102, Article ID: 157203.

[25] Gopinath, S.C.B., Awazu, K. and Fujimaki, M. (2012) Waveguide-Mode Sensors as Aptasensors. MDPI Sensors, 12, 2136-2151. http://dx.doi.org/10.3390/s120202136

[26] Fert, A. (2008) Origin, Development, and Future of Spintronics (Nobel Lectures). Reviews of Modern Physics, 80, 1517-1530. http://dx.doi.org/10.1103/RevModPhys.80.1517 
[27] Fert, A. (2008) Origin, Development, and Future of Spintronics (Nobel Lectures). Physics—Uspekhi, 51, 1336-1348 [Uspekhi Phizicheskikh Nauk (Moscow), 178, 1336-1348].

[28] Chappert, C. and Kim, J.-V. (2008) Metal Spintronics: Electronics Free of Charge. Nature Physics, 4, 837-838. http://dx.doi.org/10.1038/nphys1122

[29] Bibes, M. and Barthélémy, A. (2008) Multiferroics: Towards a Magnetoelectric Memory. Nature Materials, 7, 425-426. http://dx.doi.org/10.1038/nmat2189

[30] Prellier, W., Singh, M.P. and Murugavel, P. (2005) The Single-Phase Multiferroic Oxides—From Bulk to Thin Film. Journal of Physics: Condensed Matter, 17, R803-R832. http://dx.doi.org/10.1088/0953-8984/17/30/R01

[31] Bichurin, M.I., Petrov, V.M., Filippov, D.A., Srinivasan, G. and Nan, S.V. (2006) Magnetoelectric Materials. Academia Estestvoznaniya Publishers, Moscow.

[32] Fetisov, Y.K., Bush, A.A., Kamentsev, K.E., Ostashchenko, A.Y. and Srinivasan, G. (2006) Ferrite-Piezoelectric Multilayers for Magnetic Field Sensors. The IEEE Sensor Journal, 6, 935-938. http://dx.doi.org/10.1109/JSEN.2006.877989

[33] Srinivasan, G. and Fetisov, Y.K. (2006) Microwave Magnetoelectric Effects and Signal Processing Devices. Integrated Ferroelectrics, 83, 89-98. http://dx.doi.org/10.1080/10584580600949105

[34] Priya, S., Islam, R.A., Dong, S.X. and Viehland, D. (2007) Recent Advancements in Magnetoelectric Particulate and Laminate Composites. Journal of Electroceramics, 19, 147-164. http://dx.doi.org/10.1007/s10832-007-9042-5

[35] Grossinger, R., Duong, G.V. and Sato-Turtelli, R. (2008) The Physics of Magnetoelectric Composites. Journal of Magnetism and Magnetic Materials, 320, 1972-1977. http://dx.doi.org/10.1016/j.jmmm.2008.02.031

[36] Ahn, C.W., Maurya, D., Park, C.S., Nahm, S. and Priya, S. (2009) A Generalized Rule for Large Piezoelectric Response in Perovskite Oxide Ceramics and Its Application for Design of Lead-Free Compositions. Journal of Applied Physics, 105, Article ID: 114108.

[37] Petrov, V.M., Bichurin, M.I., Laletin, V.M., Paddubnaya, N. and Srinivasan, G. (2003) Modeling of Magnetoelectric Effects in Ferromagnetic/Piezoelectric Bulk Composites. Proceedings of the 5th International Conference on Magnetoelectric Interaction Phenomena in Crystals, MEIPIC-5, Sudak, 21-24 September 2003. http://arxiv.org/abs/cond-mat/0401645

[38] Harshe, G., Dougherty, J.P. and Newnham, R.E. (1993) Theoretical Modelling of 3-0/0-3 Magnetoelectric Composites. International Journal of Applied Electromagnetics in Materials, 4, 161-171.

[39] Chu, Y.H., Martin, L.W., Holcomb, M.B. and Ramesh, R. (2007) Controlling Magnetism with Multiferroics. Materials Today, 10, 16-23. http://dx.doi.org/10.1016/S1369-7021(07)70241-9

[40] Schmid, H. (1994) Magnetic Ferroelectric Materials. Bulletin of Materials Science, 17, 1411-1414. http://dx.doi.org/10.1007/BF02747238

[41] Ryu, J., Priya, S., Uchino, K. and Kim, H.-E. (2002) Magnetoelectric Effect in Composites of Magnetostrictive and Piezoelectric Materials. Journal of Electroceramics, 8, 107-119. http://dx.doi.org/10.1023/A:1020599728432

[42] Fang, D., Wan, Y.-P., Feng, X. and Soh, A.K. (2008) Deformation and Fracture of Functional Ferromagnetics. ASME Applied Mechanics Review, 61, Article ID: 020803.

[43] Sihvola, A. (2007) Metamaterials in Electromagnetics. Metamaterials, 1, 2-11. http://dx.doi.org/10.1016/j.metmat.2007.02.003

[44] Hill (Spaldin), N.A. (2000) Why Are There So Few Magnetoelectric Materials? Journal of Physical Chemistry B, 104, 6697-6709.

[45] Smolenskii, G.A. and Chupis, I.E. (1982) Ferroelectromagnets. Soviet Physics Uspekhi, 25, 475-493. http://dx.doi.org/10.1070/PU1982v025n07ABEH004570

[46] Ribichini, R., Cegla, F., Nagy, P.B. and Cawley, P. (2010) Quantitative Modeling of the Transduction of Electromagnetic Acoustic Transducers Operating on Ferromagnetic Media. IEEE Transactions on Ultrasonics, Ferroelectrics, and Frequency Control, 57, 2808-2817. http://dx.doi.org/10.1109/TUFFC.2010.1754

[47] Thompson, R.B. (1990) Physical Principles of Measurements with EMAT Transducers. In: Mason, W.P. and Thurston, R.N., Eds., Physical Acoustics, Volume 19, Academic Press, New York, 157-200. http://dx.doi.org/10.1016/b978-0-12-477919-8.50010-8

[48] Hirao, M. and Ogi, H. (2003) EMATs for Science and Industry: Noncontacting Ultrasonic Measurements. Kluwer Academic, Boston. http://dx.doi.org/10.1007/978-1-4757-3743-1

[49] van Suchtelen, J. (1972) Product Properties: A New Application of Composite Materials. Philips Research Reports, 27, 28-37.

[50] van den Boomgaard, J., Terrell, D.R., Born, R.A.J. and Giller, H.F.J.I. (1974) In-Situ Grown Eutectic Magnetoelectric Composite-Material. 1. Composition and Unidirectional Solidification. Journal of Materials Science, 9, 1705-1709. http://dx.doi.org/10.1007/BF00540770 
[51] van Run, A.M.J.G., Terrell, D.R. and Scholing, J.H. (1974) In-Situ Grown Eutectic Magnetoelectric CompositeMaterial. 2. Physical Properties. Journal of Materials Science, 9, 1710-1714. http://dx.doi.org/10.1007/BF00540771

[52] van den Boomgaard, J., van Run, A.M.J.G. and van Suchtelen, J. (1976) Piezoelectric-Piezomagnetic Composites with Magnetoelectric Effect. Ferroelectrics, 14, 727-728. http://dx.doi.org/10.1080/00150197608236711

[53] Annigeri, A.R., Ganesan, N. and Swarnamani, S. (2006) Free Vibrations of Simply Supported Layered and Multiphase Magneto-Electro-Elastic Cylindrical Shells. Smart Materials and Structures, 15, 459-467. http://dx.doi.org/10.1088/0964-1726/15/2/027

[54] Aboudi, J. (2001) Micromechanical Analysis of Fully Coupled Electro-Magneto-Thermo-Elastic Multiphase Composites. Smart Materials and Structures, 10, 867-877. http://dx.doi.org/10.1088/0964-1726/10/5/303

[55] Ramirez, F., Heyliger, P.R. and Pan, E. (2006) Free Vibration Response of Two-Dimensional Magneto-Electro-Elastic Laminated Plates. Journal of Sound and Vibration, 292, 626-644. http://dx.doi.org/10.1016/j.jsv.2005.08.004

[56] Wang, B.-L. and Mai, Y.-W. (2007) Applicability of the Crack-Face Electromagnetic Boundary Conditions for Fracture of Magnetoelectroelastic Materials. International Journal of Solids and Structures, 44, 387-398. http://dx.doi.org/10.1016/j.ijsolstr.2006.04.028

[57] Liu, T.J.C. and Chue, C.-H. (2006) On the Singularities in a Bimaterial Magneto-Electro-Elastic Composite Wedge under Antiplane Deformation. Composite Structures, 72, 254-265. http://dx.doi.org/10.1016/j.compstruct.2004.11.009

[58] Zakharenko, A.A. (2012) On Wave Characteristics of Piezoelectromagnetics. Pramana-Journal of Physics (Indian Academy of Science), 79, 275-285. http://dx.doi.org/10.1007/s12043-012-0308-3

[59] Wang, Y.-Z., Li, F.-M., Huang, W.-H., Jiang, X., Wang, Y.-S. and Kishimoto, K. (2008) Wave Band Gaps in Two-Dimensional Piezoelectric/Piezomagnetic Phononic Crystals. International Journal of Solids and Structures, 45, 4203-4210. http://dx.doi.org/10.1016/j.ijsolstr.2008.03.001

[60] Melkumyan, A. (2007) Twelve Shear Surface Waves Guided by Clamped/Free Boundaries in Magneto-Electro-Elastic Materials. International Journal of Solids and Structures, 44, 3594-3599. http://dx.doi.org/10.1016/j.ijsolstr.2006.09.016

[61] Zakharenko, A.A. (2010) Propagation of Seven New SH-SAWs in Piezoelectromagnetics of Class $6 \mathrm{~mm}$. LAP LAMBERT Academic Publishing GmbH \& Co. KG, Saarbruecken-Krasnoyarsk, 84 p.

[62] Zakharenko, A.A. (2011) Seven New SH-SAWs in Cubic Piezoelectromagnetics. LAP LAMBERT Academic Publishing GmbH \& Co. KG, Saarbruecken-Krasnoyarsk, 172 p.

[63] Al'shits, V.I., Darinskii, A.N. and Lothe, J. (1992) On the Existence of Surface Waves in Half-infinite Anisotropic Elastic Media with Piezoelectric and Piezomagnetic Properties. Wave Motion, 16, 265-283. http://dx.doi.org/10.1016/0165-2125(92)90033-X

[64] Zakharenko, A.A. (2012) Twenty Two New Interfacial SH-Waves in Dissimilar PEMs. LAP LAMBERT Academic Publishing GmbH \& Co. KG, Saarbruecken-Krasnoyarsk, 148 p.

[65] Zakharenko, A.A. (2012) Thirty Two New SH-Waves Propagating in PEM Plates of Class $6 \mathrm{~mm}$. LAP LAMBERT Academic Publishing GmbH \& Co. KG, Saarbruecken-Krasnoyarsk, 162 p.

[66] Auld, B.A. (1990) Acoustic Fields and Waves in Solids. 2nd Edition, Volumes I and II (Set of Two Volumes), Krieger Publishing Company, Malabar, 878 p.

[67] Dieulesaint, E. and Royer, D. (1980) Elastic Waves in Solids: Applications to Signal Processing. John Wiley, New York and Chichester, $511 \mathrm{p}$.

[68] Lardat, C., Maerfeld, C. and Tournois, P. (1971) Theory and Performance of Acoustical Dispersive Surface Wave Delay Lines. Proceedings of the IEEE, 59, 355-364. http://dx.doi.org/10.1109/PROC.1971.8177

[69] Nye, J.F. (1989) Physical Properties of Crystals. Their Representation by Tensors and Matrices. Clarendon Press, Oxford, $385 \mathrm{p}$.

[70] Newnham, R.E. (2005) Properties of Materials: Anisotropy, Symmetry, Structure (Kindle Edition). Oxford University Press Inc., Oxford and New York, 391 p.

[71] Gulyaev, Y.V. (1998) Review of Shear Surface Acoustic Waves in Solids. IEEE Transactions on Ultrasonics, Ferroelectrics, and Frequency Control, 45, 935-938. http://dx.doi.org/10.1109/58.710563

[72] Bleustein, J.L. (1968) A New Surface Wave in Piezoelectric Materials. Applied Physics Letters, 13, 412-413. http://dx.doi.org/10.1063/1.1652495

[73] Gulyaev, Y.V. (1969) Electroacoustic Surface Waves in Solids. Soviet Physics Journal of Experimental and Theoretical Physics Letters, 9, 37-38.

[74] Zakharenko, A.A. (2013) Peculiarities Study of Acoustic Waves’ Propagation in Piezoelectromagnetic (Composite) Materials. Canadian Journal of Pure \& Applied Sciences, 7, 2459-2461. 
[75] Zakharenko, A.A. (2013) New Nondispersive SH-SAWs Guided by the Surface of Piezoelectromagnetics. Canadian Journal of Pure \& Applied Sciences, 7, 2557-2570.

[76] Zakharenko, A.A. (2014) Some Problems of Finding of Eigenvalues and Eigenvectors for SH-Wave Propagation in Transversely Isotropic Piezoelectromagnetics. Canadian Journal of Pure \& Applied Sciences, 8, 2783-2787.

[77] Kiang, J. and Tong, L. (2010) Nonlinear Magneto-Mechanical Finite Element Analysis of Ni-Mn-Ga Single Crystals. Smart Materials and Structures, 19, Article ID: 015017.

[78] Zakharenko, A.A. (2011) Analytical Investigation of Surface Wave Characteristics of Piezoelectromagnetics of Class 6 mm. ISRN Applied Mathematics, 2011, Article ID: 408529.

[79] Wang, B.L., Mai, Y.-W. and Niraula, O.P. (2007) A Horizontal Shear Surface Wave in Magnetoelectroelastic Materials. Philosophical Magazine Letters, 87, 53-58. http://dx.doi.org/10.1080/09500830601096908

[80] Liu, J.-X., Fang, D.-N. and Liu, X.-L. (2007) A Shear Horizontal Surface Wave in Magnetoelectric Materials. IEEE Transactions on Ultrasonics, Ferroelectrics, and Frequency Control, 54, 1287-1289. http://dx.doi.org/10.1109/TUFFC.2007.388

[81] Zakharenko, A.A. (2015) On Existence of Eight New Interfacial SH-Waves in Dissimilar Piezoelectromagnetics of Class 6 mm. Meccanica, 50, 1923-1933. http://dx.doi.org/10.1007/s11012-015-0210-4 